proactive measures. We recommend longer placements for medical students in mental health settings for at least 4 weeks or longer. Medical schools should also promote research, discussions, and seminars on different psychiatric illnesses in order to enhance awareness among the students.

\section{Psychiatric liaison referrals and the 4PM Rush}

Kaj Svedberg ${ }^{1}$

${ }^{1}$ Rawalpindi Medical University

doi: 10.1192/bjo.2021.956

Aims. Referrals to the psychiatric Liaison team in A\&E seem to come in the afternoon in kismet as the day shift is ending. This study looked at the timing distribution of referrals to try improve amount of jobs being handed over to the evening shifts.

Method. Referrals made to Homerton University Hospital (HUH) psychiatric liaison was parsed into 1 hour bins and plotted as a histogram (data between August 2016-October 2019. $\mathrm{N}=14182$ ). The data were compared to diurnal human body temperature variation, as well as data published on Hospital Accident \& Emergency Activity 2019-20 (digital.nhs.uk) for Ambulance attendances.

Result. Referrals to HUH liaison team appear to closely follow the average human body temperature variations per hour (Pearson Correlation coefficient $=0.90)$. A peak appears to occur around $4 \mathrm{PM}$, and a low at 7AM. The referrals data also mirrored timings of official Hospital Episode Statistics (HES) reports 2019-2020 for ambulance attendance in England (Pearson Correlation coefficient $=0.94$ ). Conclusion. Attendance to $\mathrm{A} \& \mathrm{E}$ and referrals to psychiatric liaison appear correlated to a circadian bound rhythm. "The 4PM referrals rush" appears to be a genuine phenomenon replicated in not only HUH mental health referrals, but general ambulance attendance throughout all of England.

The body temperature analogue for circadian rhythm may be humorously applied here to correlate with the increased referral rates to A\&E; the emergency department could be said to be truly heating up in the afternoon. Indeed temperature and activity has already been shown to link strongly via the Arrhenius equation in cricket activity such as chirps per minute.

The conclusions drawn here are that acute mental health attendances, like general health attendances as a whole follow underlying but powerful patterns, and provisions might best be allocated to address this rather than thinking of fixed 9-5 working schedules.

\section{Service Evaluation}

Do core psychiatry trainee cognitive behaviour therapy cases meet training needs? An evaluation of core psychiatry trainee delivered CBT cases in Sheffield: implications for training and services

\author{
Dasal Abayaratne ${ }^{1 \star}$, Russell Birkett ${ }^{2}$ and John Davies ${ }^{1}$ \\ ${ }^{1}$ Sheffield Health and Social Care NHS Foundation Trust and \\ ${ }^{2}$ University of Sheffield, Sheffield Health and Social Care NHS \\ Foundation Trust \\ ${ }^{\star}$ Corresponding author.
}

doi: 10.1192/bjo.2021.804

Aims. This evaluation aims to understand if Cognitive Behaviour Therapy (CBT) cases for Core Psychiatry Trainees (CPTs) in Sheffield provide good training in therapy skills and if these can be integrated into general psychiatric practice.
Background. Completion of psychotherapy cases part of the curriculum for CPTs, with cognitive behavior therapy being one of the common modalities used. Whilst there is evidence that trainees often provide competent therapy it is unclear what cases are appropriate and how these contribute to wider CPT learning objectives. Method. CPTs who had completed a clinical case in CBT at a tertiary psychotherapy service were identified. All were surveyed and patient demographics and outcomes also collated.

Result. The results showed a significant impact on trainees understanding of CBT, applying theory to clinical context, and changed future practice. Despite being complex, $64 \%$ of patients needed no further therapy and $42 \%$ were discharged from mental health services.

Conclusion. The evaluation demonstrates the positive outcomes for patients, trainees, future clinical practice, and a move towards collaboration as laid out in the Five-year forward view for mental health. This suggests that medical trainees have a valuable contribution, and role despite minimal experience in CBT.

\section{Are we meeting local and national guidelines for} physical health assessment following admission to the meadows?

Yasmin Abbasi*, Ruairidh Morgan and Alice O’Docherty

Surrey and Borders Partnership NHS Foundation Trust

${ }^{*}$ Corresponding author.

doi: 10.1192/bjo.2021.805

Aims. We audited practice at the Meadows Inpatient Unit regarding physical health assessment, against standards set by Surrey and Borders Partnership and NICE.

Background. SABP policy states that within 24 hours of admission to inpatient services, physical health assessment should be offered. It should be completed within 72 hours. Refusal should be documented.

These guidelines state that within 2 weeks of admission blood tests should be completed, and for specific individuals an ECG should be performed.

NICE guidelines state that "physical healthcare needs" should be discussed with newly admitted patients. NICE guidelines regarding physical health monitoring for individuals with psychosis or schizophrenia recommend that assessment includes "full physical examination to identify physical illness".

NICE suggests use of antipsychotics for individuals with dementia who have severe distress, or are at risk of harming themselves or others. Those with behavioural and psychological symptoms of dementia (BPSD) should therefore be physically assessed to ensure safe use of antipsychotics may be implemented.

Method. All admissions to The Meadows over seven months were audited retrospectively. The clinical notes were accessed from Systm1.

Consensus medical opinion was reached that full examination should include: GCS/level of consciousness, cardiorespiratory, abdominal and neurological examinations.

Age, gender, diagnosis and prescriptions of psychotropic medication at time of admission were recorded.

The sample included 35 patients.

Result. $55 \%$ of patients had a diagnosis of dementia.

$63.8 \%$ of patients were prescribed antipsychotics on admission, more than other psychotropic medication. This may reflect that the most common diagnosis was dementia, commonly with associated BPSD.

$97 \%$ of patients had a physical examination completed within 24 hours; most excluded neurological examination. 91\% of patients had blood tests completed in two weeks, with the most commonly excluded tests being lipids and glucose. $86 \%$ of 
patients had an ECG in two weeks. In general, documentation of reason for not completing an examination was completed.

Conclusion. We found good compliance with recommendations for physical health assessment. Areas for improvement include better assessment of neurology and more thorough blood tests.

Recommended physical health examination for new admissions is not outlined in SABP policy. We recommend the following:

GCS/level of consciousness, cardiovascular, respiratory, abdominal, and neurological examinations, and baseline observations.

ECG should be a requirement of admission. In order to facilitate this, staff need to be trained to perform ECGs.

NICE guidelines refer to HBAlc rather than glucose, which should be reflected in SABP policy.

\section{Basic clinical equipment for physical health assessment in mid Essex inpatient units}

Hesham Abdelkhalek*, Tamsin Griffiths, Kim Motley, Raisa Ramjan and Sophia Harrop

Essex Partnership University NHS Foundation Trust ${ }^{\star}$ Corresponding author.

doi: 10.1192/bjo.2021.806

Aims. It is trust policy that the Basic Clinical Equipment for Physical Health Assessment should be available on each unit. The standard for this audit is therefore $100 \%$ completion.

Background. This was a cross-sectional study of six mental health units across Mid Essex. We audited equipment and consumables in comparison to trust policies. For the purpose of the audit we designed an audit tool.

Method. Overall compliance across all wards for all audited items was $77.5 \%(64.9 \%-87.5 \%)$. Average compliance for equipment provision $83.3 \%(73.9 \%-91.3 \%)$ was greater than that for consumables $72.1 \%(58.8 \%-82.4 \%)$.

Result. When looking at the compliance on each unit separately, our data show that no unit has met the standard of $100 \%$ for equipment or consumables. From all units, one of the two older adults' inpatient units had the highest overall compliance and highest compliance for consumables at $87.5 \%$ and $82.4 \%$ respectively while the perinatal unit had the lowest overall compliance and lowest compliance for consumables at $64.9 \%$ and 58.8 respectively. For the equipment compliance, intensive care unit and one of the older adults tied for the highest compliance at 91.3\% while male inpatient unit and perinatal inpatient unit were tied the lowest compliance at $73.9 \%$.

Conclusion. This is an audit to assess the availability of Basic Clinical Equipment for Physical Health Assessment on inpatient units in Mid Essex. With an audit standard of $100 \%$ completion, it shows that overall compliance on all units was $77.5 \%$ which is not meeting our standard.

\section{Audit cycle - VTE risk assessment in inpatient wards in mid Essex}

Hesham Abdelkhalek*, Adel Elagawany, Fiona McDowall, Matthew Leahy, Emily Baker and Nkechi Penberton

Essex Partnership University NHS Foundation Trust ${ }^{\star}$ Corresponding author.

doi: 10.1192/bjo.2021.807

Aims. It is trust policy that the VTE risk assessment should be completed for every patient admitted to wards. The standard for this audit is therefore $100 \%$ completion. We completed the audit in October 2018 and closed the loop in September 2019. Method. This was a cross-sectional study of all patients on all the wards according to patients' list on the electronic system (Paris) on certain date. In the first audit we used an audit tool from a similar audit performed in another area in the trust. For the purpose of re- audit we designed an audit tool to reflect the changes made in the electronic form.

Result. In the re-audit, there was noticeable improvement in the completion rate compared to initial audit (95\% vs. $82 \%$ ); however, there was still under-performance. An interesting observation of the re-audit is that $74 \%$ percent of admissions had VTE risk assessments forms completed on same day of admission or next day compared to only $45 \%$ in previous audit.

Conclusion. When looking at the completion of individual components on the VTE forms there are still some room for improvement as well. For example, in $26 \%$ of the patients there was no documentation about the use of prophylactic anticoagulants before admission compared to $34 \%$ in our previous audit. Also in $7 \%$ of the patients there was no documentation about the outcome of the assessment compared to only $3 \%$ in previous audit.

This is an audit to assess the completion of electronic VTE forms as per trust policy. Following the initial audit we made recommendations to improve completion rate. In the re-audit there was an improvement in total completion rate but we have not met the goal of $100 \%$ yet.

\section{'Comfortable, safe and valued': an analysis of the impact of COVID-19 on Hertfordshire's Community Perinatal Team}

\section{Stephanie Adeyemi* and Sarah Cohen}

Hertfordshire Partnership University NHS Foundation Trust ${ }^{\star}$ Corresponding author.

\section{doi: $10.1192 /$ bjo.2021.808}

Aims. This study aimed to assess the impact that the COVID-19 pandemic has had on the Hertfordshire Community Perinatal Team (CPT) group interventions and the innovations made.

Background. The CPT is a multidisciplinary mental health service that runs three groups: Circle of Security $(\mathrm{CoS})$, Emotional Coping Skills (ECS) and a peer support group - Wellbeing and Lifestyle. The service has received an increase in referrals during the COVID-19 pandemic.

Method. Methods: Team member and client semi structured interviews were conducted with answers transcribed in real time and analysed. Patient clinical records were accessed via PARIS and analysed in order to identify patient demographics within each group and whether these had changed during the pandemic. Clinical outcome measures and client feedback were evaluated to see whether the change in groups is impacting their clinical effectiveness.

Result. Results: Innovations made by the CPT include: groups becoming virtual, launching of the new Circle of Security Group which helps women tackle the 'Ghosts in the Nursery' and strengthen maternal bonds, restructuring existing groups, breakout room forums and incorporating communication platform apps such as Whatsapp. The Wellbeing and Lifestyle Group increased in size and reach (7 women from 7 areas in 2019 vs 12 women from 12 areas in 2021) with an increased retention rate (71\% in 2019 vs $100 \%$ in 2021) and a decreased attrition rate $(29 \%$ in 2019 to $0 \%$ in 2021). The Emotional Coping Skills group experienced similar changes (10 areas represented in 2019 vs 15 different areas in 2021) with an increased 